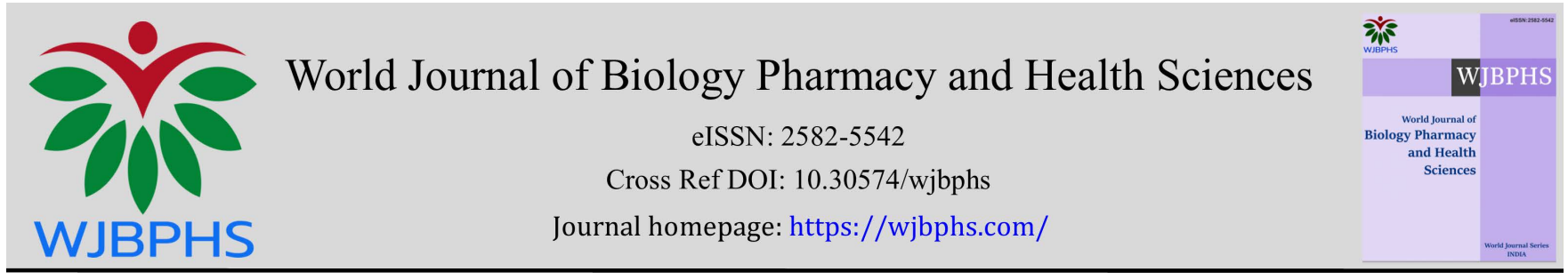

(RESEARCh ARTicle)

\title{
Management of pre-analytical nonconformities at the biochemistry laboratory in Antananarivo in 2019
}

\author{
Miora Koloina Ranaivosoa 1, *, Valdo Rahajanirina 2, Zafindrasoa Domoina Rakotovao Ravahatra 3, \\ Jaquinot Randriamora ${ }^{4}$, Olivat Rakoto Alson, Andry Rasamindrakotroka ${ }^{5}$ \\ ${ }^{1}$ Biologist. Laboratory of Biochemistry of Joseph Ravoahangy Andrianavalona University Hospital Antananarivo, \\ Madagascar. \\ ${ }^{2}$ Medical Biology Student, Laboratory of Biochemistry of Joseph Ravoahangy Andrianavalona University Hospital \\ Antananarivo, Madagascar. \\ ${ }^{3}$ Biologist, Laboratory Unit of University Hospital Center Joseph Raseta Befelatanana Antananarivo, Madagasca. \\ ${ }^{4}$ Médical student, Faculty of Medicine of Antananarivo, Madagascar \\ 5 Professor of Biological Haematology, Medical Biology Department of the Faculty of Medicine Antananarivo, \\ Madagascar. \\ ${ }^{6}$ Professor of Immunology. Laboratory of Training and Research in Medical Biology, University of Antananarivo, \\ Madagascar.
}

World Journal of Biology Pharmacy and Health Sciences, 2021, 05(02), 006-011

Publication history: Received on 23 January 2021; revised on 31 January 2021; accepted on 03 February 2021

Article DOI: https://doi.org/10.30574/wjbphs.2021.5.2.0010

\begin{abstract}
Management of pre analytical nonconformities within a laboratory is a critical step in ensuring the reliability of results. The objectives of this study are to evaluate the non-compliance of the pre-analytical phase at the Paraclinical Training and Biochemistry Research Unit of the Joseph Ravoahangy Andrianavalona University Hospital Center, to describe in detail the state of play and the progress of this stage. This is a retrospective descriptive study over a period of 5 months from November 01, 2018 to March 31, 2019 within the Paraclinical Training and Biochemistry Research Unit of Joseph Ravoahangy Andrianavalona University Hospital Center. All patient files recorded during this study period have been exploited. Only inpatient records were included in this study. In this study, 5, 71\% of pre-analytical non-conformities were recorded. The most frequent non-conformities (recorded 248 times that means $56.88 \%$ of the whole nonconformities) were related to the swab or its container, followed by non-conformities related to the prescription sheet (recorded 96 times, that means 22.02\%). Pre-analytical non-conformities were the most frequently identified in the surgical intensive care department with 25.24\%, followed by the medical service (17.92\%). Most of the nonconformities observed were due to preventable human error. However, the laboratory must know how to control nonconformities in order to prevent them and ensure the quality of the analyses.
\end{abstract}

Keywords: Antananarivo; Biochemistry; Nonconformity; Pre-analytical

\section{Introduction}

Non-compliance (NC) is any deviation from standards, practices, procedures, regulations, which could directly or indirectly cause injury or illness, damage to the priority, to the workplace environment or a combination of these elements. [1]. Management of test NC within a laboratory is a critical step in ensuring the reliability of results. The objectives of this study are to evaluate the NC of the pre-analytical phase at the Paraclinical Training and Biochemistry

\footnotetext{
${ }^{*}$ Corresponding author: Miora Koloina Ranaivosoa

Laboratory of Biochemistry of Joseph Ravoahangy Andrianavalona University Hospital Antananarivo, Madagascar. 
Research Unit (UPFR) of the Joseph Ravoahangy Andrianavalona University Hospital Center (CHU-JRA), to describe in detail the state of play and the progress of this stage.

\section{Materials and methods}

This is a retrospective descriptive study over a period of 5 months from November 01, 2018 to March 31, 2019 within the UPFR Biochemistry of CHU-JRA. All patient files recorded during this study period have been exploited. Only inpatient records were included in this study. The parameters studied were age, gender, types of non-compliance, prescribing services. Data were entered into Microsoft Office Excel ${ }^{\circledR} 2013$ and processed by Epi Info ${ }^{\circledR} 7.0$ software.

\section{Results}

\subsection{Overall pre-analytical NC}

The number of requests for biochemical analysis processed during the study period from hospital departments was 7629. One or more NC were found out of 436 requests (5.71\%).

\subsection{Distribution of the different types of NC}

The most frequent NC (recorded 248 times that means $56.88 \%$ of the whole NC) were related to the swab or its container, followed by NC related to the prescription sheet (recorded 96 times, that means 22.02\%). (Table I)

Table I Distribution of the different types of nonconformities.

\begin{tabular}{|l|c|c|}
\hline Types of NC & Number & Percentage \\
\hline NC related to swab identification & 6 & $1.38 \%$ \\
\hline NC related to the prescription sheet & 96 & $22.02 \%$ \\
\hline NC related to the swab and its container & 248 & $56.88 \%$ \\
\hline NC related to the transport conditions of the swab & 69 & $15.82 \%$ \\
\hline NC relating to health and safety conditions & 17 & $3.90 \%$ \\
\hline Total & 436 & $100 \%$ \\
\hline
\end{tabular}

\subsection{1. $N C$ related to the swab and its container}

NC related to the swab and its container were the most frequent pre-analytical abnormalities in our study $248 / 436$, i.e., $56.88 \%$. The missing tube in relation to the analysis request represented the most frequent NC in this type of noncompliance, 112 times or $45.16 \%$ (93 urine vials, 14 grey blood glucose tubes and 5 biological fluid samples not reaching the laboratory). Haemolysis sampling was also a fairly frequent cause of NC observed in the UPFR Biochemistry of CHUJRA with a rate of $43.55 \%(108 / 248)$. NC between the requested analysis and the tube received at the laboratory was noted on 20 requests (8.06\%) and the insufficiency in quantity or incorrectly filled tube was recorded 8 times (3.23\%).

\subsubsection{NC related to the prescription sheet}

In relation to the prescription sheet or the analysis request form, 39 (i.e. $40.63 \%$ ) NC related to the absence of the doctor's stamp or the identity of the prescribing doctor were recorded.

The absence of the date and time of sampling on the analysis request form was found on 20 prescriptions (20.83\%), the absence of information on the department prescribing the request in third position with a rate of $15.63 \%$, the absence of clinical information in fourth position with a rate of $13.54 \%$ and finally the absence of patient identification (age and/or gender and/or name) and the inadequacy of the prescribed analysis represented respectively $8.33 \%$ and $1.04 \%$ of all non-compliances in relation to the prescription sheet.

\subsection{3. $N C$ related to the transport conditions of the swab}

NC associated with the transport conditions of the swab represented $15.82 \%$ of all NC. For this type of NC, delayed transmission of the swab was found at $100 \%$, no routing error was recorded $(0 \%)$. 


\subsection{4. $N$ C relating to health and safety conditions}

NC relating to health and safety conditions were among the least frequent pre-analytical anomalies ( 6 cases recorded). The tube soiled with blood or other biological fluid was recorded 3 times (50\%) as well as the prescription sheet soiled with blood or other biological fluid (50\%).

\subsection{5. $N$ C related to swab identification}

$\mathrm{NC}$ related to swab identification were the least observed pre-analytical abnormalities.

Lack of tube labelling was recorded only twice (33.33\%). The NC between the identification on the prescription sheet and the one mentioned on the sample was found on 4 requests (i.e. $66.66 \%$ ).

\subsection{Proportion of pre-analytical NC according to the prescribing department}

Pre-analytical non-compliances were the most frequently identified in the surgical intensive care department with $25.24 \%$, followed by the medical service (17.92\%), emergency departments $(12.74 \%)$, pediatrics (10.61\%), medical resuscitation (9.43\%), Befelatanana maternity services (9.67\%), reception desk and emergency services (ATU) of Joseph Raseta Befelatanana Hospital (7 , 07\%), surgery (3.30\%), oncology (2.83\%), ophthalmology (0.48\%), maxillofacial surgery of Befelatanana $(0.23 \%)$ and other departments $(0.39 \%)$ (figure 1$)$.

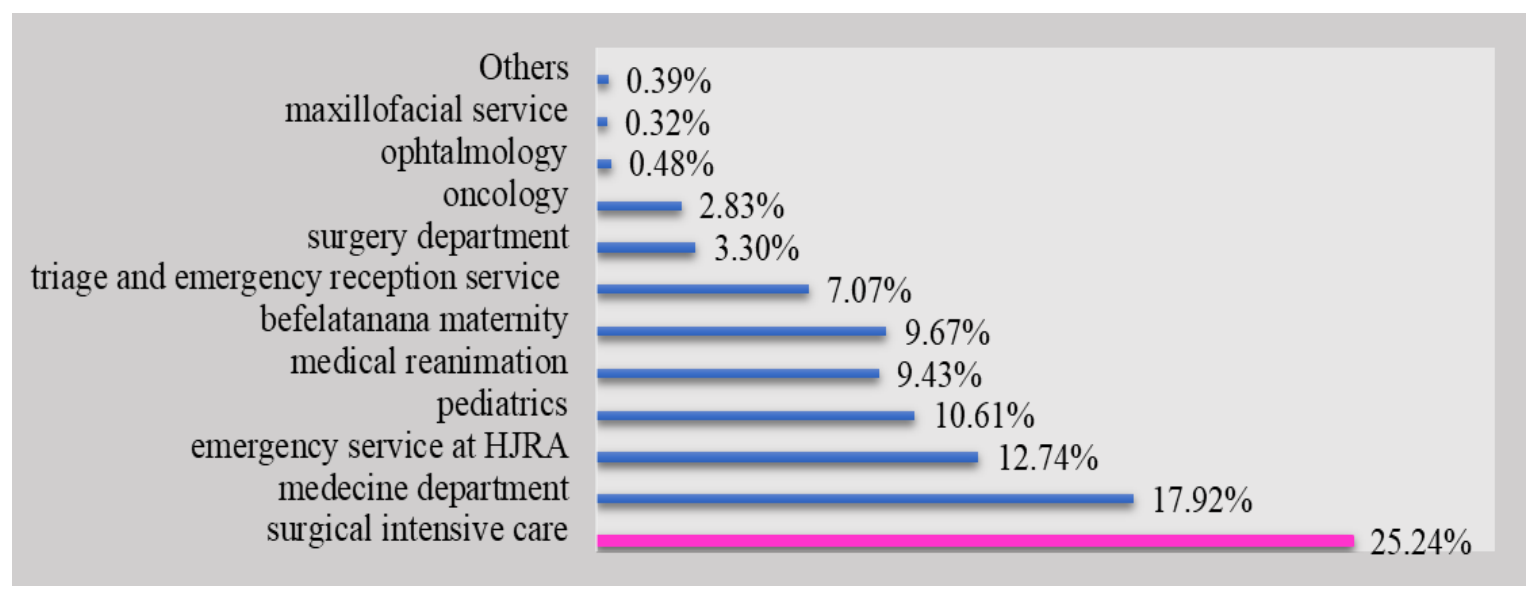

Figure 1 Proportion of pre-analytical NC according to the prescribing department

\section{Discussion}

\subsection{Overall pre-analytical NC}

In this study, 436 requests had one or more NC among 7629 requests, or $5.71 \%$.

In Casablanca, in 2017, a study on the management of pre-analytical NC at the Biochemistry Laboratory of CHU lbn Rochd was able to find 489 pre-analytical NC in 58317 requests, this means $0.83 \%$. This rate is lower than the rate in our study [2].

On the other hand, in Rabat, in 2011, 12015 cases of pre-analytical non-compliance were listed at the biochemistry laboratory of Mohamed V Military Hospital of Instruction among the 30321 requests. This corresponds to a slightly higher rate of $39.63 \%$ [3].

Pre-analytical errors detected can have an impact on the results of analysis or can be the cause of inconsistencies or even erroneous diagnoses and inappropriate therapies; this is a concern for all medical biology laboratories, and for the biologist mainly in terms of the quality of his services [1]. 


\subsection{Distribution of the different types of NC}

\subsection{1. $N C$ related to the swab and its container}

According to the results of the present study, non-compliances related to the swab and its container were the most observed type of non-compliance with $56.88 \%$ of the cases $(248 / 436)$ of which $45.16 \%(112 / 248)$ corresponded to tubes that did not reach the laboratory. The latter were due to an omission to send or to carry out the collection procedure or the urine collection procedure. In these cases, the tests will not be carried out and the patient is obliged to come to the laboratory a second time. This generates discomfort for the patient and a delay in his or her therapeutic management, but also causes discomfort for the biologist who feels confused and responsible.

The hemolyzed samples represented $43.55 \%$ (that is to say $108 / 248$ ), followed by the mismatch between analysis and the tube that reached the laboratory with $8.06 \%$ (that is to say 20/248) and finally the poorly filled tubes with $3.23 \%$ $(8 / 248)$.

These different types of NC are grouped by some authors as unusable swaps, which in this study were then found at $26.46 \%$ compared to the overall NC (136/514). In Rabat, in 2011, 2357 cases of pre-analytical NC related to unusable swabs were recorded at the biochemistry laboratory of the Mohamed V Military Hospital of Instruction in the 30855 requests: corresponding to a slightly lower rate of $7.63 \%$ [3].

Among the unusable swabs, hemolyzed samples were observed in 43.55\% (108/248).

This NC can have a very deleterious effect on patient care and the reputation of a laboratory because of its impact on test results. It is important to note that several biochemical parameters have been affected by hemolysis [4-7].

This high rate of hemolyzed swabs in our study could be explained by the increasing use of syringes in clinical departments to take specimen from patients.

After hemolyzed swabs, insufficient volumes and poor tube selection accounted for 3.23\% and $8.06 \%$ respectively. In other studies, such as that of Annaix V et al. in Bailleul in 2011, 32.6\% insufficient volumes and 11.3\% poor tube selection were found [8]. Poor tube selection is most often linked to a lack of knowledge of the nature of the appropriate anticoagulant and the absence of laboratory sampling manuals.

\subsection{2. $N C$ related to the prescription sheet}

In relation to the prescription sheet or the analysis request form, 39 (i.e. $40.62 \%$ ) NC related to the absence of the doctor's stamp or the identity of the prescribing doctor were recorded. The absence of information about the original department was found in $15.62 \%$ of cases. In order to be considered compliant, the prescription form must be correctly signed by the prescribing physician and the prescribing department must be mentioned. In the event of a pathological result that is life-threatening and requires emergency treatment, the laboratory staff immediately informs the prescribing physician or the prescribing department after verification and control of the results.

The absence of sampling date and time on the analysis request form was found on 20 prescriptions (20.83\%). The precision of the sampling time is important, it allows the biologist to consider a bad conservation. In fact, biological swabs are living tissues within which metabolism continues. They are fragile environments.

The absence of clinical information was found in fourth place with a rate of $13.54 \%$. It is crucial to know precisely the context in which the examination was prescribed. Thus, every biologist should be informed about the clinical context in order to interpret the results of biological analyses and ensure that they are consistent with the observed situation. This guarantees a judicious interpretation of the results by making it possible to check their consistency with the various factors that may interfere. These factors may be related to physiological or pathological variations, to the environment, or to drug use $[9,10]$.

Lack of patient identification was found in $8.33 \%$ of cases. Laboratory staff are often confronted with medical examination sheets that are either incomplete or with illegible handwriting. Lack of identification may concern the first and last name, age, gender. However, all information about the identification and the disease are essential for the interpretation of the results because of physiological and pathological variations. 


\subsubsection{NC related to transport conditions}

Delayed transmission of the swab was found at $100 \%$ in this type of NC, no routing error was recorded ( $0 \%)$.

The delivery of swabs to the laboratory must meet criteria and comply with recommendations to ensure proper storage of the biological samples collected, to preserve the safety of personnel and to protect them from accidents involving exposure to blood. The time of transport and the conditions under which the sample is transported may affect the quality and quantity of the constituents of the biological samples [1,11]. Prolonged contact of plasma or serum with the figurative elements of the blood may alter the concentration of the metabolites to be determined [12].

\subsubsection{NC relating to health and safety conditions}

NC with health and safety conditions was among the least frequent pre-analytical abnormalities (6 cases recorded). Staining of tubes and/or sheets with blood is often observed when the swab collector completes the quantity of the defective tube by adding part of a blood sample collected on another tube, without knowing formally and precisely the foreseeable consequences of such a practice for the patient as well as for each of the intervening parties (swab collector and laboratory staff). It is recommended that all specimens be considered potentially infectious.

\subsubsection{NC related to swab identification}

Six cases of NC related to swab identification, i.e. 1.38\% (6/436), were observed for inpatients, including 2 cases $(33.33 \%)$ without tube labelling and 4 cases $(66.66 \%)$ of discrepancy between identification on the examination request sheet and that on the sample. The identification of patients during blood collection in the care departments remains too often poorly controlled, whereas an error at this stage can have serious consequences. One possible explanation is that health care workers have multiple tasks in their departments that cause oversights and errors. This could also be explained by the lack of training of health care staff on the importance of this step.

Indeed, tube identification is a key step allowing the biologist to link the biological sample to the patient, differentiate homonyms, link the patient to his or her history and assign appropriate reference values (man $\neq$ woman $\neq$ child) [3].

\subsection{Proportion of pre-analytical NC according to the prescribing department}

In this study, pre-analytical NC was most frequently found in the surgical intensive care department with $25.24 \%$. This could be explained by the frequency of requests for samples from this department and also because the HJRA Hospital is a surgical hospital where our laboratory is located. In another study, non-compliance of tests was much more observed in the pediatrics department [13].

\section{Conclusion}

In this study entitled: "Management of pre-analytical non-conformities at the Biochemistry laboratory in Antananarivo in 2019", 5.71\% of pre-analytical NC were recorded. Most of the NC observed were due to preventable human error. However, the laboratory must know how to control NC in order to prevent them and ensure the quality of the analyses.

\section{Compliance with ethical standards}

\section{Acknowledgments}

We would like to thank all the staff of the laboratory of University Hospital of Ravoahangy Andrianavalona and all the laboratory technicians. Similarly, we would like to express our gratitude to the director of establishment for authorizing us to carry out this study.

\section{Disclosure of conflict of interest}

The Author declare no conflict of interest.

\section{References}

[1] Bouhamdi K. Descriptive aspect of the non-conformities of the pre-analytical phase in the HMIMV virology laboratory.Doctorat en Pharmacie. Rabat:Université Mohamed V Faculté de Médecine et de Pharmacie. 2020. 
[2] El Aouni M, Arji S, El Kettani A, Anibat N, Kamal N. Management of pre-analytical non-conformities at the Biochemistry laboratory.17èmes journées marocaines de Biologie Clinique. 2017.

[3] Benziane N. Management of the preanalytical phase in the HMIMV Biochemistry laboratory.Doctorat en Pharmacie.Rabat:Université Mohamed V Faculté de Médecine et de Pharmacie. 2011.

[4] Young DS. Effects of Preanalytical Variables on Clinical Laboratory Tests, 2nd Edition. AACC press. 1997.

[5] Melvin RG, Kenneth WR, Sheila AJ. Graphical Comparisons of Interferences in Clinical Chemistry Instrumentation. Clinical Chemistry. 1986; 3: 470-5.

[6] Ranaivosoa MK, Rahajanirina V, Rasamoelina M., Rakotoniaina T, Rakoto Alson O, Rasamindrakotroka A. Hemolysis influence on the analytical performances of the automaton Bs 300 in the dosage of 5 parameters. International Journal of Development Research.2019; 9(12): 32176-80.

[7] Ranaivosoa MK, Ramaminiaina ME, Rakotoniaina T, Rahajanirina V, Rasamoelina T, Randriamanantany ZA, Rakoto Alson 0, Rasamindrakotroka A. Influence of hemolysis on the analytical performance of BS 300 in the determination of 6 enzymes. International Journal For Research In Biology \& Pharmacy. 2020; 5(2): 2208-93.

[8] Annaix V, Rogowski J, Joyau M, Jaouën E. Management of non-conformities in the biology laboratorymedical: application to non-conformities of samples for the year 2009.Ann Biol Clin. 2011; 69(3): 363- 72.

[9] Drosdowksy S.C. Factors to be taken into account for the blood collection for the establishment reference values. Ann.Biol.Clin. 1980; 38: 251-60.

[10] Siest G, Henny J, Schiele F. References in clinical biology. Elsevier,ed.Collection Option Bio. 1990; 679.

[11] Duchassaing D, Elalamy I, Michotey 0 et Piemont Y. Quality assurance of the pre-analytical phase: sorting centers.Revue Française des Laboratoires. 1998; 299: 29-36.

[12] Duchassaing D. Pre-analytical phase in biochemistry: Quality control process. Revue Française des Laboratoires. 1999; 317: 27-34.

[13] Jaouad M, Lemrani A, Katfy K, Zerouali K, Belabbes H, Mdaghri N. El. Management of non-Conformitie preanalytical at the Microbiology Laboratory of the Ibn Rochd-Casablanca University Hospital. Journal de Biologie Médicale. 2018; 7: 227-9. 